\title{
AVALIAÇÃO DO DESENVOLVIMENTO NEUROPSICOMOTOR EM CRIANÇAS DE 0-18 MESES ACOMPANHADAS EM UMA UNIDADE BÁSICA DE SAÚDE DA FAMÍLIA
}

\section{Ana Ravenna Sales Soares}

Programa de Residência Multiprofissional em Saúde da Família e Comunidade da Residência Integrada em Saúde da Escola de Saúde Pública do Ceará (RIS-ESP/ CE), Brasil.

\section{Edna Jéssica Lima Gondim}

Fisioterapeuta, pela Universidade Federal do Ceará (UFC); Mestranda em Saúde da Mulher e da Criança, pela UFC, Brasil.

\section{Gisele Maria Melo Soares Arruda}

Fisioterapeuta graduada, pela Faculdade Estácio do Ceará. Doutoranda em Saúde Coletiva, pela Universidade Federal do Ceará, Brasil.

\section{Thiago Brasileiro de Vasconcelos}

Fisioterapeuta, pela Faculdade Estácio do Ceará. Doutorando em Farmacologia, pela Universidade Federal do Ceará. Centro Universitário Católica de Quixadá, Brasil.

E-mail: thiagobvasconcelos@hotmail.com
RESUMO: Avaliar o desenvolvimento neuropsicomotor de crianças de 0-18 meses de determinada microárea assistida por uma Unidade Básica de Saúde da Família (UBASF). Realizou-se um estudo descritivo, observacional e longitudinal com estratégia de análise quantitativa dos resultados em uma UBASF localizada na cidade de Maracanaú/CE. Para coleta de dados, utilizou-se a ficha de avaliação presente na Caderneta de Saúde da Criança (CSC) agregada a outra ficha elaborada pelos autores onde continham dados sociodemográficos. Participaram do estudo 17 crianças. Foi possível evidenciar que nove (53\%) crianças apresentaram todos os marcos esperados para a idade de acordo com a tabela de acompanhamento do desenvolvimento presente na CSC. Das crianças que não apresentaram todos os marcos para a faixa etária, sete $(87,5 \%)$ estavam entre um a seis meses e um $(12,5 \%)$ entre 13 a 18 meses. Entretanto, nenhuma criança foi considerada com atraso no desenvolvimento, pois estavam dentro dos períodos específicos para realizar cada marco. Evidenciou-se que nenhuma criança apresentou atraso no desenvolvimento neuropsicomotor. Por fim, ressalta-se a importância da realização da triagem completa durante as consultas de puericultura e da utilização do instrumento já presente na CSC.

PALAVRAS-CHAVE: Desenvolvimento infantil; Saúde da criança; Atenção integrada às doenças prevalentes na infância; Estratégia saúde da família; Saúde pública.

\section{EVALUATION OF NEUROPSYCHOMOTOR DEVELOPMENT IN 0-18-MONTH CHILDREN IN A FAMILY BASIC HEALTH UNIT}

\begin{abstract}
The neuropsychomotor development of 0-18-monthold children of a micro-area attended by a family basic health unit in Maracanau CE Brazil, is evaluated by a descriptive, observational and longitudinal study. Data were collected by the assessment of the Children's Health Sheet coupled to another form with sociodemographic data prepared by the authors. Seventeen children participated in the study. Nine (53\%) children had all the marks expected for their age according to the follow-up development table. Seven $(87.5 \%)$ out of the children, who did not present all the marks, were aged between 1 and 6 months, and one child (12.5\%) was aged between 13 and 18 months. No child had development delays since they were all within the specific periods for each mark. No child had any delay in neuropsychomotor development. Results reveal the importance of complete selection during puericulture attendance and the employment of the CHS.
\end{abstract}

KEY WORDS: Child development; Child's health; Integrated Care to Diseases Prevalence in Childhood; Family health Strategy; Public Health. 


\section{INTRODUÇÃO}

O Sistema Único de Saúde (SUS) definiu como estratégia para o cuidado integral e direcionado às necessidades de saúde da população, a organização de Redes de Atenção à Saúde (RAS). As RAS são arranjos organizativos das ações e serviços de saúde de diferentes densidades tecnológicas e missões assistenciais com o objetivo de complementar a atenção à saúde com base no território. Dentre os diversos pontos das RAS, destaca-se a atenção básica como coordenadora do cuidado e porta de entrada preferencial do sistema, com base no trabalho interprofissional, na adscrição de clientela e na oferta de um cuidado generalista e longitudinal (BRASIL, 2012).

A Estratégia Saúde da Família (ESF), por meio de relevante expansão de cobertura populacional, vem aprimorando cada vez mais o acesso da população às ações de saúde, ao passo que se consolida como um dos eixos estruturantes do SUS e da Atenção Básica brasileira (BRASIL, 2009). O atendimento de puericultura operacionaliza-se, nessa estratégia, para o acompanhamento da saúde das crianças, objetivando verificar o crescimento e desenvolvimento, assegurar a cobertura vacinal, estimular a prática do aleitamento materno, orientar a introdução da alimentação complementar e prevenir as doenças mais frequentes no primeiro ano de vida (LEITE, 2003).

O desenvolvimento infantil tem seu início ainda na vida intrauterina e sofre influências de diversos fatores biológicos e ambientais. Quando a criança nasce, tornase essencial o monitoramento do desenvolvimento psicomotor, comunicação, funções sensoriais, cognição e funcionamento sócio-adaptativo, pois a partir destes poderão ser detectados os fatores de risco para transtornos do neurodesenvolvimento e realizar as respectivas estimulações precoces (ZEPPONE et al., 2012; CENTERS FOR DISEASE CONTROL AND PREVENTION, 2014).

Tendo em vista que o acompanhamento do Desenvolvimento Neuropsicomotor (DNPM) é importante para o crescimento saudável da criança, fazem-se necessários mais estudos que possam avaliar o comportamento do desenvolvimento motor e a aplicabilidade do instrumento presente na caderneta.
A relevância deste estudo está em poder oferecer à comunidade científica, como também à sociedade, mais uma fonte de pesquisa que tem como base a prevenção, promoção e/ou recuperação de agravos no DNPM nas crianças acompanhadas na atenção básica.

Sabendo disto, o presente estudo teve por objetivo avaliar o DNPM de crianças de 0 - 18 meses de uma microárea assistida por uma Unidade Básica de Saúde da Família (UBASF) no município de Maracanaú/ CE.

\section{MÉTODOS}

Estudo descritivo, observacional e transversal, com estratégia de análise quantitativa dos resultados, realizado na UBASF Elias Boutala Salomão, situada no município de Maracanaú/CE.

A coleta de dados ocorreu no período de novembro de 2016 a fevereiro de 2017. Conforme aprovação do projeto pelo comitê de ética e pesquisa da Escola de Saúde Pública do Ceará (Parecer no: 2.200.378), foram selecionadas, de uma determinada microárea da UBASF, todas as crianças recém-nascidas que realizam atendimento programado/continuado de puericultura, com idade entre 0 e 18 meses, independente do gênero ou etnia, e que os pais e/ou responsáveis aceitaram participar da pesquisa mediante assinatura do termo de consentimento livre e esclarecido. Foram excluídas as crianças que não retornaram para as consultas de puericultura ou mudaram de endereço e, consequentemente, de UBASF.

Durante as consultas de puericultura na UBASF, ocorreu um atendimento compartilhado entre a enfermagem e a fisioterapia com os pacientes da microárea 51. A enfermeira realizava seu atendimento de costume avaliando peso, altura, perímetro cefálico, e fazendo orientações gerais sobre a saúde da criança. Já a fisioterapeuta abordou sobre a avaliação do DNPM e importância de estimular desde cedo o desenvolvimento da criança para que a mesma adquira autoconfiança, autoestima e desenvolva a capacidade de se relacionar bem com outras crianças, com a família e com a comunidade.

Para coleta de dados, utilizou-se o instrumento de vigilância do desenvolvimento presente na Caderneta 
de Saúde da Criança (BRASIL, 2015); o qual indica na linha superior a idade da criança em meses; e, na coluna correspondente, as habilidades que devem ser testadas para a idade (destacadas em amarelo) e, por fim, é registrado como marco presente $(\mathrm{P})$, marco ausente (A) ou marco não verificado $(\mathrm{NV})$.

Esta caderneta é considerada um documento importante para acompanhar a saúde, o crescimento e o desenvolvimento da criança, desde nascimento até os nove anos de idade. Ela é dividida em duas partes, a primeira é dedicada aos cuidadores da criança, contendo informações e orientações sobre os cuidados com a saúde da criança. Já a segunda parte é destinada aos profissionais de saúde, contendo fichas para registro de informações importantes, gráficos de crescimento, instrumento de vigilância do DNPM e tabelas para registros das vacinas aplicadas (BRASIL, 2015).

Os dados sociodemográficos (nome da criança, sexo, nome da mãe, data de nascimento da criança, número de consultas de pré-natal, tipo de parto, endereço e número do cartão do SUS) foram coletados por meio de uma ficha elaborada pelos autores.

\section{RESULTADOS}

$\mathrm{Na}$ análise sociodemográfica, foi verificado que a maioria das crianças é do sexo feminino (70,6\%), reside na região metropolitana de Fortaleza $(76,5 \%)$ e possui cartão do SUS $(82,4 \%)$ (Tabela 1). Com relação à faixa etária, 11 (66\%) crianças apresentaram idade entre um e seis meses, e três (17\%) de sete a 12 meses, dados que se repetem para 13 e18 meses.

Com relação à raça/cor/etnia das crianças avaliadas, os responsáveis responderam que oito $(47,1 \%)$ eram consideradas pardas, seis $(35,3 \%)$ brancas e três $(17,6 \%)$ negras (Tabela 1). Também foi avaliada a assiduidade das mães no período pré-natal e constatou-se que $11(64,7 \%)$ compareceram a mais de seis consultas.
Tabela 1. Dados sociodemográficos da população estudada

\begin{tabular}{ccc}
\hline Itens & N & $\%$ \\
\hline Sexo & & \\
Masculino & 05 & 29,4 \\
Feminino & 12 & 70,6 \\
Faixa etária & & \\
$01 — 06$ meses & 11 & 66 \\
07 -12 meses & 03 & 17 \\
13 18 meses & 03 & 17 \\
& & \\
Etnia & & 35,3 \\
Branca & 06 & 17,6 \\
Negra & 03 & 47,1 \\
Parda & 08 & \\
Nascimento & & 76,5 \\
Maracanaú & 13 & 23,5 \\
Fortaleza & 04 & \\
Consultas pré-natal & & 64,7 \\
Acima de 06 & 11 & 11,8 \\
Menor que 06 & 02 & 23,5 \\
Não lembro & 04 & 82,4 \\
Cartão SUS & & 17,6 \\
Sim & 14 & \\
Não & 03 & \\
\hline
\end{tabular}

Ao avaliar o período de início do pré-natal, observou-se que dez $(58,8 \%)$ iniciaram o pré-natal no primeiro trimestre, seguido por duas $(11,8 \%)$ que afirmaram ter iniciado no segundo trimestre (Tabela 2).

Tabela 2. Dados relacionados ao inicio do pré-natal

\begin{tabular}{lcc}
\hline Início do pré-natal & $\mathbf{N}$ & $\%$ \\
\hline $1^{\circ}$ trimestre & 10 & 58,8 \\
$2^{\circ}$ trimestre & 02 & 11,8 \\
$3^{\circ}$ trimestre & 01 & 5,9 \\
Não lembra & 04 & 23,5 \\
\hline
\end{tabular}

Quanto à gestação e ao parto, 15 (88,2\%) crianças foram geradas por meio de gravidez simples e duas $(11,8 \%)$ em gravidez múltipla; 16 (94,1\%) nasceram no hospital e uma (5,9\%) nasceu em domicílio; dez (58,8\%) nasceram em parto normal, sete (41,2\%) em parto cesáreo e nenhuma de parto a fórceps (Figura 1). 


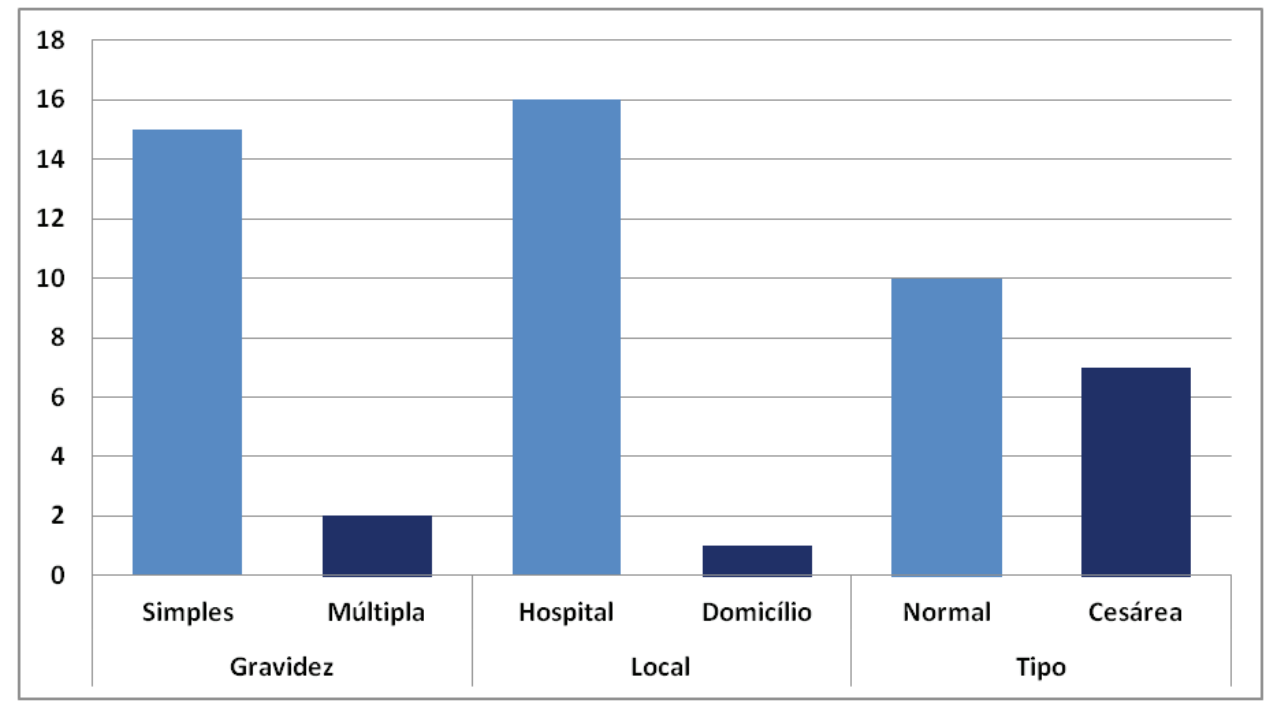

Figura 1. Perfil gestacional e tipo de parto da população estudada

Constatou-se que todas as crianças realizaram o teste do pezinho, porém quatro $(23,5 \%)$ receberam o resultado e 13 (76,5\%) não receberam; dos recebidos uma obteve resultado considerado alterado. Já para o teste do ouvido, apenas três $(17,6 \%)$ realizaram; dos realizados duas não receberam e uma foi considerado normal.

Os dados obtidos com o instrumento de vigilância do desenvolvimento, presente na caderneta da criança, foram os seguintes: das crianças avaliadas nove (53\%) apresentaram todos os devidos marcos para a idade de acordo com a tabela de acompanhamento do desenvolvimento presente na Caderneta de Saúde da Criança e oito (47\%) não apresentaram algum dos marcos esperados para a idade.

Ao analisar os dados das oito crianças que não apresentaram todos os marcos, pode-se observar que seis (75\%) eram do sexo feminino, sete $(87,5 \%)$ com idade de um a seis meses e uma (12,5\%) 13 a 18 meses.

O dado que se apresentou como mais alterado, em sete crianças $(87,5 \%)$ foi o item "segurar objetos", em que o avaliador deve oferecer um objeto tocando no dorso da mão ou dedos da criança e a mesma deverá abrir as mãos e segurar o objeto pelo menos por alguns segundos. Porém não deve ser considerado atrasado, pois são apresentados no segundo mês de vida e a criança tem até o quarto mês para apresentar esse marco no desenvolvimento. Ademais, salienta-se que nenhuma criança apresentou essa alteração após o quarto mês de vida.
Outros dados alterados foram: uma criança $(12,5 \%)$ que não apresentou os itens "constrói torre de 02 cubos" (observar se a criança consegue colocar 01 cubo sobre o outro sem que ele caia ao retirar sua mão) e "fala 03 palavras" (observar se, durante o atendimento, a criança diz pelo menos 03 palavras que não sejam nomes de membros da família ou de animais de estimação; considerar a informação do acompanhante). Entretanto, assim como os dados anteriores, não são considerados atrasos, pois foram apresentados no $16^{\circ}$ mês e a criança tem até o $18^{\circ}$ para apresentar estes marcos.

\section{DISCUSSÃO}

$\mathrm{O}$ atendimento compartilhado é uma tecnologia leve, que facilita e potencializa a integralidade do cuidado ao paciente, além de promover uma troca de saberes e experiências aos que trabalham na Atenção Básica (MELLO FILHO; SILVEIRA, 2005; BRASIL, 2014). Nesse sentido, o presente estudo realizou-se por meio da prática de atendimentos compartilhados/consulta conjunta/ interconsulta, conforme previsto na metodologia do apoio matricial proposta no processo de trabalho do NASF (BRASIL, 2014). Tal experiência, lamentavelmente, não é um hábito adotado entre a ESF e o NASF; entretanto, durante a residência em saúde da família, os profissionais residentes executaram ações desse tipo e mostraram a sua importância aos demais funcionários da UBASF. 
Com a finalidade de garantir a qualidade da assistência prestada à criança, o Ministério da Saúde propõe a realização de pelo menos sete consultas de puericultura no primeiro ano e meio de vida. Usualmente, tal prática é realizada pelo enfermeiro e pelo médico (LONDRINA, 2006). É importante observar este desenvolvimento nos primeiros anos de vida, pois é nesta fase que o tecido nervoso mais cresce e amadurece, estando, assim, susceptível aos agravos. Além disso, outro fator importante é a plasticidade do tecido nervoso neste período, facilitando assim as respostas emitidas pelas crianças aos estímulos recebidos do meio ambiente ou das intervenções, quando necessárias (BRASIL, 2015).

Recentemente, a Secretaria de Saúde do Estado do Ceará publicou uma nota técnica na qual alerta sobre os casos de atraso do DNPM e destaca que, dentre os principais fatores associados ao atraso, percebese que um fato atual se encaixa nos anteriores: as arboviroses (dengue, Zika e febre Chikungunya). Entre as principais manifestações neurológicas, com história prévia de infecção por arboviroses, estão a encefalite, meningoencefalite, mielite, paralisia flácida aguda, encefalomielite disseminada aguda, síndrome de GuillainBarré e microcefalia (CEARÁ, 2016). A microcefalia tem sido associada à infecção pelo vírus Zika (ZIKV), que é transmitido pela picada de mosquito Aedes aegypti. Entretanto, recentemente relatou-se a transmissão vertical de ZIKV da mãe para o feto, infecção esta que, pode ter ocorrido intraútero ou durante parto (SCHULER-FACCINI et al., 2016).

Nesse sentido, Shonkoff, Boyce e Mcewen (2009) destacam que o alicerce para o sucesso na escola e, futuramente, no ambiente de trabalho e comunidade são as habilidades de cognição e linguística, saúde física, saúde emocional e interação social que se desenvolvem nos primeiros anos de vida. A avaliação do DNPM é de suma importância para a detecção dos riscos e/ou atrasos no desenvolvimento de forma prática e padronizada, especialmente no cenário de grande incidência de arboviroses (DORNELAS, DUARTE; MAGALHÃES, 2015). Porém, estudos revelam que os atrasos no DNPM infantil são de origem multifatorial (BISCEGLI et al., 2007; SACCANI et al., 2007; HALPERN et al., 2008; LIMA; LIMA, 2012).
Dornelas, Duarte e Magalhães (2015) confirmam esse fato apontando os principais fatores associados ao atraso, que vão da concepção, gestação e parto; fatores ambientais, familiares e socioeconômicos; causas neurológicas e até má nutrição, o que pode ocasionar atrasos no desenvolvimento, seja de forma permanente ou transitória. Porém, mesmo com toda essa importância, há poucos estudos nacionais que apresentam essa temática da vigilância do desenvolvimento de crianças e seus desfechos (ZEPONNE; VOLPON; CIAMPO, 2012; CUSTÓDIO; CREPALDI; CRUZ, 2012).

Segundo o Relatório Final da Conferência sobre Determinantes Sociais da Organização Mundial de Saúde

Investir nos primeiros anos de vida é uma das medidas que permitirá, com maior probabilidade, a redução das desigualdades em saúde, no espaço de uma geração [...] O desenvolvimento da primeira infância, em particular o desenvolvimento físico, socioemocional e linguístico-cognitivo, determina de forma decisiva as oportunidades na vida de uma pessoa e a possibilidade de gozar de boa saúde, já que afeta a aquisição de competências, a educação e as oportunidades de trabalho (OMS, 2008).

Entretanto, deve-se ressaltar que, no Brasil, não há testes normorreferenciados para parâmetros de avaliação infantil (HALPERN et al., 2008; RAMOS; MORAIS, 2011). Segundo Lima, Cavalcante e Costa (2016), por este fato, as alterações no DNPM podem passar despercebidas, evidenciando-se apenas quando a criança já se encontra em idade escolar, o que justifica uma necessidade de avaliar precocemente a fim de minimizar os danos e atrasos infantis.

Desde 1986, várias ações sobre o desenvolvimento infantil vêm sendo implementadas pela área da saúde, uma delas foi o Programa de Atenção Integral à Saúde da Criança (PAISC), que enfatizou o acompanhamento do crescimento e desenvolvimento, considerado o eixo da assistência à criança. Entretanto, pelas elevadas taxas de mortalidade infantile do perfil de adoecimento das crianças na época, as ações de vigilância do crescimento foram priorizadas, e isso se mantém até os dias atuais no âmbito da atenção básica. Destaca-se a existência de instrumentos para a vigilância do crescimento e desenvolvimento, por 
meio da caderneta de saúde da criança. Contudo, os estudos sobre a utilização e preenchimento da caderneta são escassos e revelam falhas consideráveis na utilização da mesma, principalmente em relação ao preenchimento dos marcos do desenvolvimento infantil (GAIVA; SILVA, 2014).

No presente estudo, pode-se observar que oito crianças não apresentaram os marcos do desenvolvimento para sua idade. A prevalência da ausência de marcos foi apresentada no segundo mês de vida, entretanto, com a detecção precoce e a intervenção junto aos familiares de como estimular o desenvolvimento de cada criança, no quarto mês as crianças apresentaram os marcos preconizados.

As reais necessidades das crianças/famílias ou as intervenções mais apropriadas não têm sido o foco de muitos profissionais de atenção básica, que geralmente, na puericultura, estão mais baseados no modelo de cuidados às doenças, nutrição e crescimento. A definição dos conhecimentos e habilidades necessárias à atuação desses profissionais seria uma estratégia para a mudança no foco dos mesmos, indo ao encontro do presente estudo (REGALADO; HALFON, 2001).

Neste estudo, outros dados como número de consultas de pré-natal, tipo de parto e localidade da moradia foram observados a fim de identificar possíveis influências no DNPM, corroborando com o estudo de Zeponne, Volpon e Ciampo (2012), que destaca a importância de se conhecer o contexto social e familiar da criança, informações sobre a gestação da mãe, principal cuidador, rotina e possíveis fatores de risco. Vale ressaltar que um recém-nascido é considerado de alto risco para atrasos no DNPM quando apresenta prematuridade, asfixia perinatal grave, baixo peso, alteraçóes neurológicas, pequeno para idade gestacional (PIG), crescimento anormal do perímetro cefálico e infecção congênita (SBP, 2000). Segundo Amorim et al. (2005), as crianças que possuem alguma das condições acima, apresentam risco maior de alterações no crescimento e no DNPM.

As UBASF's, segundo a Política de Humanização do Pré-Natal e Nascimento, devem garantir o acesso e a qualidade no acompanhamento pré-natal, oferecendo assistência médica, exames laboratoriais, remédios, vacinas e tratamentos necessários (BRASIL, 2014). No presente estudo, observou-se que 64,7\% das mães compareceram às seis consultas mínimas preconizadas pelo Ministério da Saúde. Supõe-se que a baixa escolaridade e o nível socioeconômico podem ter sido fator que contribuiu para que $35,3 \%$ não tenham comparecido as seis consultas e/ ou não lembram a quantidade exata de consultas.

O teste da Orelhinha é um exame para detectar precocemente, até o sexto mês de vida, a perda auditiva congênita e/ou adquirida no período neonatal; é justificado pelo fato de as crianças com perdas auditivas terem maiores dificuldades para desenvolvimento neuropsicomotor (BRASIL, 2014). Segundo Hilú e Zeigelboim (2007), o aparelho auditivo está formado e funciona a partir do sexto mês gestacional. Prevenir a perda da função auditiva se faz necessária e importante para reduzir os efeitos sobre a função da linguagem e o desenvolvimento neuropsicomotor.

Entretanto, fica constatada neste estudo a deficiência na realização desse teste, provavelmente por conta do município pesquisado não realizar este exame. Sendo assim, os pacientes são encaminhados para Fortaleza, e, na maioria das vezes, os mesmos não podem se deslocar até a capital, deixando de realizar o exame.

Já com relação ao teste do pezinho, a taxa de realização foi de $100 \%$, corroborando com o Manual de Normas Técnicas e Rotinas Operacionais do Programa Nacional de Triagem Neonatal do Ministério da Saúde (BRASIL, 2004). Todavia, observa-se que nem todos receberam o resultado. Constatando-se, assim, uma falha no sistema, pois o exame é realizado, porém há grande demora na entrega dos resultados, o que atrapalha nas investigações e prognósticos.

$\mathrm{O}$ presente estudo encontrou algumas dificuldades, como o curto período para executar a coleta de dados; disponibilidade de agenda tanto da fisioterapia como da enfermagem; e o tamanho da amostra. Porém diversos fatores foram observados, por exemplo, a necessidade de se utilizar a tabela de avaliação do desenvolvimento neuropsicomotor presente na caderneta da criança, a eficácia do atendimento compartilhado, com intensa troca de saberes e experiências, e a presença dos marcos nos atendimentos subsequentes. 


\section{CONCLUSÃO}

A puericultura consiste numa diretriz ideal para acompanhamento integral do crescimento e desenvolvimento infantil. Neste estudo, nenhuma criança apresentou atraso no DNPM, porém essa triagem completa não é hábito durante as consultas, visando mais o crescimento e o desenvolvimento de forma superficial. É importante que os profissionais utilizem o instrumento presente na caderneta da criança, a fim de se obter mais resultados e possibilitar discussões para a melhora da saúde da população infantil brasileira.

Destaca-se também sobre a importância de avaliar as crianças e orientar os pais e/ou responsáveis sobre a maneira de estimular o desenvolvimento, pois ficou constatado que as crianças que foram avaliadas e não apresentaram os marcos devidos para a idade foram estimuladas em casa a partir das orientações recebidas, e, na consulta seguinte, apresentaram seus devidos marcos de acordo com o instrumento de vigilância do desenvolvimento.

\section{REFERÊNCIAS}

AMORIM, R. H. C. et al. Acompanhamento do recémnascido de risco. Temas de neuropediatria. Belo Horizonte: [s.n], 2005.

BISCEGLI, T.S.; POLIS, L. B.; SANTOS, L. M.; VICENTIN, M. Avaliação do estado nutricional e do desenvolvimento neuropsicomotor em crianças frequentadoras de creche. Rev Paul Pediatr., v. 25, n. 4, p. 337-342, 2007.

BRASIL. Ministério da Saúde. Cadernos de Atenção Básica. Secretaria de Atenção à Saúde, Departamento de Atenção Básica. Brasília, DF: Ministério da Saúde, 2014.

BRASIL. Ministério da Saúde. Humanização do parto e do nascimento. Brasília, DF: Ministério da Saúde, 2014. (Cadernos Humaniza SUS, v. 4).

BRASIL. Ministério da Saúde. Secretaria de Atenção à Saúde. Departamento de Ações Programáticas Estratégicas. Coordenação-Geral de Saúde da Criança e Aleitamento Materno. Caderneta de saúde da criança: menino: passaporte da cidadania. 10. ed. Brasília; Ministério da Saúde, 2015. 92p.
BRASIL. Ministério da Saúde. Secretaria de Atenção à Saúde. Departamento de Atenção Básica. Política Nacional de Atenção Básica. Brasília, DF: Ministério da Saúde, 2012.

BRASIL. Ministério da Saúde. Secretaria de Atenção à Saúde. Departamento de Atenção Básica. Saúde da criança: nutrição infantil - aleitamento materno e alimentação complementar. Brasília, DF: Ministério da Saúde, 2009.

BRASIL. Ministério da Saúde. Secretaria de Atenção à Saúde. Departamento de Atenção Especializada. Manual de normas técnicas e rotinas operacionais do Programa Nacional de Triagem Neonatal. Brasília, DF: Ministério da Saúde, 2004. (Série A. Normas e Manuais Técnicos).

BRASIL. Ministério da Saúde. Secretaria de Políticas de Saúde. Departamento de Atenção Básica. Saúde da criança: acompanhamento do crescimento $\mathrm{e}$ desenvolvimento infantil. Brasília, DF: Ministério da Saúde, 2002. 100 p. (Série Cadernos de Atenção Básica; n. 11).

CEARÁ (Estado). Secretaria de Saúde do Ceará. Nota técnica: vigilância das manifestações neurológicas do Ceará. Ceará, 2016.

CENTER FOR DISEASE CONTROL. Prevalence of autism spectrum disorder among children aged 8 years: autism and developmental disabilities monitoring network. United States, 2010. Morbidity and Mortality Weekly Report Surveillance Summary, v. 63, n. 2, p. 1-22, 2014.

CUSTÓDIO, Z. A. O.; CREPALDI, M. A.; CRUZ, R. M. Desenvolvimento de crianças nascidas pré-termo avaliado pelo teste de Denver-II: revisão da produção científica brasileira. Psicol Reflex Crit (Porto Alegre), v. 25, n. 2, p. 400-406, 2012.

DORNELAS, L. F.; DUARTE, N. M. C.; MAGALHÃES, L. C. Atraso do desenvolvimento neuropsicomotor: mapaconceitual, definições, usos e limitações do termo. Rev Paul Pediatr., v. 33, n. 1, p. 88-103, 2015.

GAIVA, M. A. M.; SILVA, F. B. Caderneta de saúde da 
criança: revisão integrativa. Revista de Enfermagem, v. 8, n. 3, p. 742-749, 2014.

HALPERN, R.; BARROS, A. J. D.; MATIJASEVICH, A.; SANTOS, I. S.; VICTORA, C. G.; BARROS, F. C. Developmental status at age 12 months according to birth weight and Family income: a comparison of two Brazilian birth cohorts. Cad Saúde pública. v. 24, n. 3, p. 444-450, 2008.

HILÚ, M. R. P. B.; ZEIGELBOIM, B. S. O conhecimento, a valorização da triagem auditiva neonatal e a intervenção precoce da perda auditiva. Rev CEFAC, v. 9, n. 4, p. 563$570,2007$.

LEITE, G. B.; BERCINI, L. O. Caracterização das crianças atendidas na puericultura do programa saúde da família do município de Campo Mourão, Paraná, em 2003. Cienc. cuid. Saúde., v. 4, n. 3, p. 224-230, 2005.

LIMA, A. K. P.; LIMA, A. O. Perfil do desenvolvimento neuropsicomotor e aspectos familiares de crianças institucionalizadas na cidade de Recife. Rev CES Psicol., v. 5, n. 1, p. 11-25, 2012.

LIMA, S. S.; CAVALCANTE, L. I. C.; COSTA, E. F. Triagem do desenvolvimento neuropsicomotor de crianças brasileiras: uma revisão sistemática da literatura. Fisioter Pesqui., v. 23, n. 3, p. 336-342, 2016.

LONDRINA. Prefeitura do Município. Autarquia Municipal de Saúde. Saúde da criança: protocolo. Londrina, PR, 2006.

MELLO FILHO, J.; SILVEIRA, L. M. C. Consulta Conjunta: uma estratégia de capacitação para a atenção integral à saúde. Revista Brasileira de Educação Médica, v. 29, n. 2, p. 147-151, 2005.

OLIVEIRA, W. K.; COELHO, G. E.; FRANÇA, G.V.A. Boletim Epidemiológico: situação epidemiológica de ocorrência de microcefalias no Brasil, 2015. Bol. Epidemiológico da SVS/MS, v. 46, n. 34, p. 1-3, 2015.

ORGANIZAÇÃO MUNDIALDA SAÚDE-OMS. Relatório da Organização Mundial da Saúde sobre determinantes sociais da saúde. Genebra: OMS, 2008.
RAMOS, A. D.; MORAIS, R. L. S. Vigilância do desenvolvimento neuropsicomotor de crianças de um programa DST/AIDS. Fisioter Pesqui., v. 18, n. 4, p. 371376, 2011.

REGALADO, M.; HALFON, N. Primary Care Services Promoting Optimal Child Development from birth to age 3 years. Archives of Pediatrics \& Adolescent Medicine, v. 155, n. 12, p. 1311-1322, 2001.

SACCANI, R.; BRIZOLA, E.; GIORDANI, A. P.; BACH, S.; RESENDE, T. L.; ALMEIDA, C. S. Avaliação do desenvolvimento neuropsicomotor em crianças de um bairro da periferia de Porto Alegre. Sci Med (Porto Alegre), v. 17, n. 3, p. 130-137, 2007.

SCHULER-FACCINI, L. et al. Possible association between ZIKV vírus infection and microcephaly - Brazil, 2015. MMWR Morb Mortal Wkly Rep., n. 65, p. 59-62, 2016.

SHONKOFF, J. P.; BOYCE, W. T.; MCEWEN, B. S. Neuroscience, molecular biology and the childhood roots of health disparities: building a new framework for health promotion and disease prevention. The Journal of the American Medical Association, v. 301, p. 22522259, 2009.

SOCIEDADE BRASILEIRA DE PEDIATRIA (SBP). Indicadores de risco para distúrbios no DNPM. 2000.

ZEPONNE, S. C.; VOLPON, L. C.; CIAMPO, L. A. D. Monitoramento do desenvolvimento infantil realizado no Brasil. Rev Paul Pediatr., 30, n. 4, p. 594-599, 2012.

Recebido em: 2017-08-04

Aceito em: 2017-12-05 